\title{
Electro-osmosis Modulated Viscoelastic Embryo Transport in Uterine Hydrodynamics: Mathematical Modelling
}

\author{
V. K. Narla \\ Department of Mathematics \\ GITAM University \\ Hyderabad-502329, India \\ Email:vknarla@gmail.com
}

\author{
Dharmendra Tripathi \\ Department of Sciences and Humanities \\ National Institute of Technology \\ Uttarakhand -246174, India \\ Email: dtripathi@nituk.ac.in
}

\author{
O. Anwar Bég \\ Aeronautical and Mechanical Engineering \\ University of Salford \\ Manchester, M54WT,UK \\ Email: gortoab@gmail.com
}

\begin{abstract}
Embryological transport features a very interesting and complex application of peristaltic fluid dynamics. Electroosmotic phenomena are also known to arise in embryo transfer location. The fluid dynamic environment in embryological systems is also known to be non-Newtonian and exhibits strong viscoelastic properties. Motivated by these applications, the present article develops a new mathematical model for simulating two-dimensional peristaltic transport of a viscoelastic fluid in a tapered channel under the influence of electro-osmosis induced by asymmetric zeta potentials at the channel walls. The robust Jeffrey viscoelastic model is utilized. The finite Debye layer electro-kinetic approximation is deployed. The moving boundary problem is transformed to a steady boundary problem in the wave frame. The current study carries significant physiological relevance with an ever-increasing desire to study intrauterine fluid flow motion in an artificial uterus. The consequences of this model may introduce a new mechanical factor for embryo transport to a successful implantation site. Hydrodynamic characteristics are shown to be markedly influenced by the electro-osmosis, the channel taper angle and the phase shift between the channel walls. Furthermore it is demonstrated that volumetric flow rates and axial flow are both enhanced when the electro-osmotic force aids the axial flow for specific values of zeta potential ratio. Strong trapping of the bolus
\end{abstract}


(representative of the embryo) is identified in the vicinity of the channel central line when the electro-osmosis opposes axial flow. The magnitude of the trapped bolus is observed to be significantly reduced with increasing tapered channel length whereas embryo axial motility is assisted with aligned electro-osmotic force.

\section{Introduction}

Peristalsis is an intriguing biological propulsion mechanism which is observed both internally and externally in nature. In internal physiological systems it features in infant heart pumping, digestion and blood flow. In external loco-motion it arises in snake crawling. Peristalsis is also fundamental to phloem translocation in plants. In all these applications the fluid or body is propelled by rhythmic muscular contractions which generate a wave of contraction along the conduit or body walls. Another complex manifestation of peristaltic propulsion is oviductal peristalsis which is critical to the conveyance of an ovum to the fertilization location. Neither the ovum nor the embryo move by self-propulsion mechanisms.Cyclic uterine peristalsis aids in the transport of sperm to the fallopian tube. Subsequent to this it also assists in the conception process. Ureteral peristalsis therefore assists significantly in the efficient delivery of human sperm via the uterus towards the fundal site for implantation (oviduct) via intrauterine fluid flow and ensures that the process is completed within the time limits of fertilization and implantation. Uterine wall motility and peristaltic pumping has therefore received substantial attention among clinical and engineering researchers. Lang et al. [1] studied the cellular mechanisms underlying the action of endogenously-released agents in ureteral peristaltic wave propagation. Ezzati et al. [2] considered normal human tubal anatomy and histology in peristaltic uterine function. Embryo hydrodynamics aspects were considered in uterine peristalsis by Yaniv et al. [3], Bulletti and de Ziegler [4], Eytan and Elad [5]. Zhu et al. [6] investigated hyper-stimulation effects on uterine peristalsis as a mode of improving fertility. Zhu et al. [7] further highlighted that uterine peristalsis post-embryo transfer can deviate substantially from pre-embryo transfer.

In parallel with these studies, significant work has also been conducted on the mathematical and computational simulation of uterine peristaltic flows. These studies deploy an extremely wide range of approaches including symbolic software and integral methods, two-dimensional finite difference algorithms, analytical methods of regularized Stokeslets and computational fluid dynamics codes. Aranda et al. [8] simulated the ovum transport with a geometrically simple peristaltic flow, mimicking the ovum as a spherical vesicle of finite volume rather than a massless point particle. They utilized the method of regularized Stokeslets to visualize the flow field and determine pressure wave characteristics. Eytan et al. [5,9] constructed a two-dimensional Stokes flow model of uterine peristalsis. These results indicate that the axial transport is largely affected by the phase shift between the walls and the tapered angle of the channel walls. They also identified that the reflux phenomenon is the cause of unsuccessful embryo implantation in women who suffer from hydrosalpinx. Yaniv et al. [3] performed computational simulations using FIDAP software with moving mesh to capture propagating walls to study the effect of the catheter which is inserted into a two-dimensional peristaltic channel. They analyzed the relative timing of initial injection with the phase of the peristaltic wave. Eytan et al. [10] performed embryo transfer simulations with a transparent laboratory model of the uterine cavity. In this model, the catheter was loaded with alternating air and colored liquid media and they suggested that the viscosity of the transferred liquid should be as close as possible to that of the uterine fluid in order to avoid transport 
of embryos towards the cervix.

The interaction of electrical fields and fluids has many forms and includes electro hydrodynamics and electrokinetics. The latter includes, electrophoresis in which solid particles (e.g. macromolecules such as proteins or nucleic acids) are transported using an electric field. Another subset of electrokinetic flows is electro-osmosis in which a liquid is moving and the support solid material may be a gel, membrane, capillary, etc. The fundamental mechanism of electro-osmotic flow (EOF) arises at the interface where the fluid solution and material attain opposite charges. This zone is defined as the electrical double layer. Under the imposition of an electrical field, the electrical double layer is mobilized by the resulting Coulomb force. The zeta potential and Debye length are critical features of EOF phenomena. EOF has also been exploited in very high precision micro-pumps in the medical, chemical and civil engineering industries [11] including soil embankment stabilization. Further applications arise in botanical systems (e.g. streaming potentials developed by pressures in large pores such as xylem or phloem vessels or cell walls) [12], shark predatory sensing mechanisms [13], hydrophobic microfluidics [14] and alternating current DNA concentrators [15]. The charge on the wall in EOF dynamic is due to the particular chemistry of the solid-fluid interface and may indeed vary along the channel owing to specific inhomogeneities of the wall biomaterial. In microfluidics the variation may be attributable to contamination of the wall by chemicals contained in the fluid stream. Numerous mathematical investigations of EOF have been reported. The linear effects associated with the electrical field effect make this area particularly attractive to mathematicians. Tripathi et al. [16] examined the unsteady electro-osmotic peristaltic pumping of blood in cylindrical vessels of finite length, using the Debye-Hckel linearization and lubrication theory to evaluate the effects of inverse Debye length and Helmholtz-Smoluchowski velocity on the axial velocity, pressure gradient, volumetric flow rate and local wall shear stress distributions. Sheu et al. [17] investigated computationally the nonlinear behavior of electro-osmotic flow in the human meridian. Ghosal [18] developed a lubrication model for electro-osmotic flow in a straight microfluidic channel of arbitrary cross-sectional geometry and distribution of wall charge. He showed analytically that the volume flux of fluid is a linear function of the applied pressure drop and electric potential drop across it and that the associated coefficients are computable in terms of the geometry and charge distribution on the wall. He further identified that owing to the axial variation in channel properties there arises an induced pressure gradient and an associated secondary flow which produces enhanced Taylor dispersion. Numerical simulation of electro-osmotic hydrodynamics in a micro-pump was reported by Bengtsson and Robinson [19] who considered high flexibility of the channel walls.

The above studies were all confined to Newtonian viscous fluids. Physiological fluids (e.g. cervical mucus, gastric liquids, sperm, intrauterine and amniotic media) are however inherently non-Newtonian [20]. Such fluids may be delineated broadly into three classes, namely "rate type", "differential type" and "integral type". Viscoelastic models accurately simulate the shear stress-strain behavior of embryological media. They fall into the "rate type" of category and can accurately model many sophisticated effects including memory, normal stress differences, relaxation, retardation, stretching, molecular reorientation etc. Many examples of excellent viscoelastic constitutive theories have been developed and employed in many areas of medical and biochemical engineering. Bég et al. [21] used a Walters-B (short memory) fluid model to simulate timedependent coupled flow and heat diffusion in permeable tissue media with the PSPICE electro-thermal software. Tripathi and Bég [22] employed the homotopy perturbation method to derive solutions for peristaltic pumping of generalized Maxwell 
viscoelastic fluids in non-deformable porous media. Norouzi et al. [23] deployed an optimized perturbation technique to compute the flow and thermal convection in a curved tube with the Reiner-Rivlin second order viscoelastic model. Another robust model which is popular in biophysics is the convected Jeffery model which is also equivalent to the Oldroyd-B model. This has the advantage that several other models can be extracted as special cases (e.g. Maxwell model, second order fluid with a normal stress coefficient and the Newtonian model). Tripathi and Bég [24] have employed the Jeffery model to study analytically the heat diffusion in peristaltic digestive transport. Alarabiet al. [25] have also used the Jeffery model to obtain homotopy perturbation solutions for the peristaltic flow through a eccentric cylindrical conduit with viscosity variation.

The current study examines electro-osmotic peristaltic pumping of a viscoelastic Jeffrey fluid in a tapered channel. In this regard it compliments and amalgamates previous studies in which only aspects of the physics have been considered. For example, Sadeghi et al. [26] analyzed viscoelastic electro-osmotic flow in a slit microchannel with constant wall heat fluxes, although they did not consider channel tapering or peristaltic wave propagation. Berli et al. [27] used a simple power-law model to analyze the electrokinetic flow of through slit and cylindrical microchannels, deriving expressions for flow rate and electric current as functions of the simultaneously applied electric potential and pressure gradients. Ferrás et al. [28] employed linear and exponential PTT viscoelastic models to derive semi-analytical solutions for the electro-osmotic annular flow of non-Newtonian liquids. They considered both no slip and slip boundary conditions. Further studies include Jiang et al. [29] who examined unsteady slip flow of fractional Oldroyd-B fluid in a circular microchannel (using Laplace and finite Hankel transforms) and Misra et al. [30] who deployed Eringen's micropolar fluid model to study electro-osmotic oscillatory blood flow in microchannels. These studies however did not address biorheological embryological electro-osmotic propulsion which is the focus of the current investigation. A detailed parametric study of the influence of taper angle, phase shift, viscoelastic material parameter, Debye length and maximum electro-osmotic velocity on volumetric flow rate, axial velocity field, bolus dynamics and pressure distribution is conducted. Computations are evaluated with regard to actual clinical implications. The current study is novel and has not appeared thus far in the scientific literature.

\section{Mathematical formulation and flow regime}

\subsection{Governing equations}

The considered model simulates peristaltic flow of an incompressible viscoelastic fluid of Jeffrey model in a microchannel, which is driven by the coupled forces electric field (electroosmosis) and asymmetric wall motility. Unsteady fluid motion with in a two-dimensional channel is induced by two sinusoidal wave trains that are propagated with constant speed $c$ along the channel's axis

$$
\begin{array}{r}
r=h_{1}(x, t)=-a-x \tan [\alpha]+\frac{b}{2}(1+\cos (k x-\omega t+\theta)) \quad(\text { Lower wall), } \\
r=h_{2}(x, t)=a+x \tan [\alpha]-\frac{b}{2}(1+\cos (k x-\omega t)) \quad(\text { Upper wall). }
\end{array}
$$




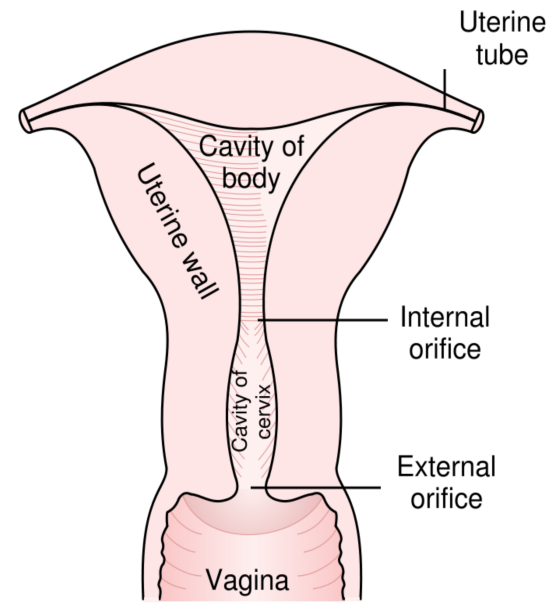

(a)

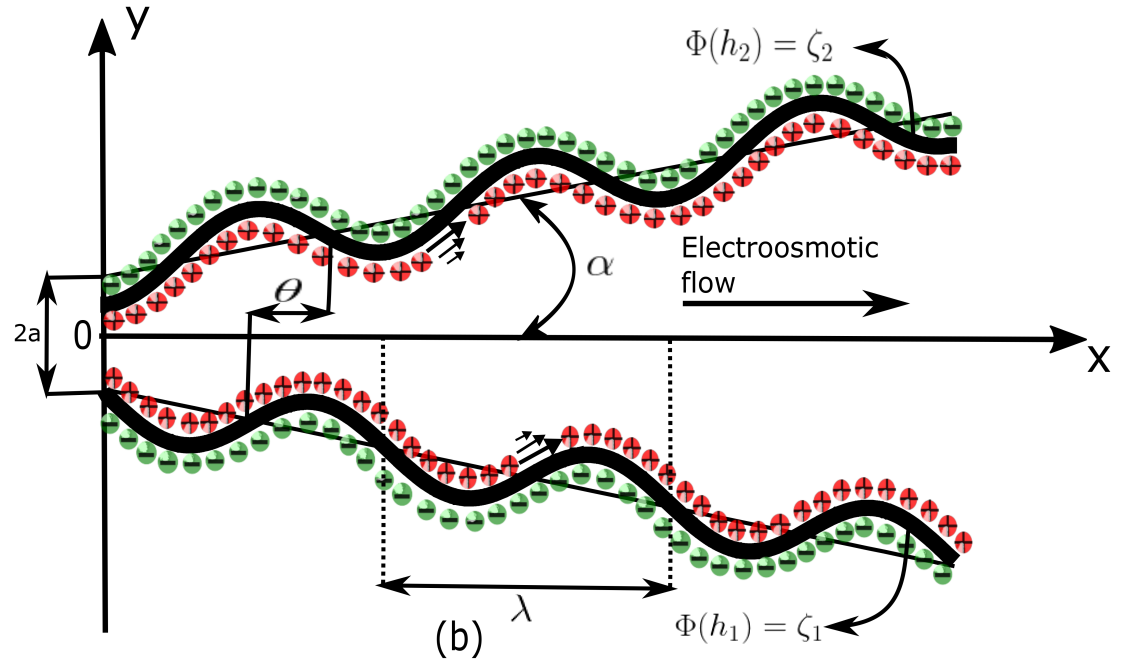

(b)

Fig. 1. (a) Human uterus wall structure; (b) Schematic view of the physical problem.

where $x$ is the dimensional axial coordinate, $t$ is the dimensional time, $\alpha$ is the inclined angle of the walls, $a$ is the channel half width at the narrowest end of the channel, $b$ is the wave amplitude, $\lambda$ is the wave length, $T$ is the wave period ( $k=2 \pi / \lambda$ and $\omega=2 \pi / T)$. The phase difference $\theta$ varies in the range $0 \leq \theta \leq \pi$, where $\theta=0$ defines symmetric wall motility. It is assumed that the wave length is large compared with the channel's width $((a+x \tan \alpha) \ll \lambda)$. The conservation equation in vectorial form for an incompressible Jeffrey fluid with electrokinetic body force, takes the form:

$$
\rho\left(\frac{\partial}{\partial t}+\mathbf{V} \cdot \nabla\right) \mathbf{V}=\nabla \cdot \mathbf{T}+F_{E}
$$

In the above equations $\mathbf{V}=u(x, r, t) \widehat{e}_{x}+v(x, r, t) \widehat{e}_{r}$ is a divergence-free velocity vector field $(\nabla \cdot \mathbf{V}=0)$ subject to the no-slip boundary conditions on the walls, $\rho$ is the fluid density, $\mathbf{T}$ is the stress tensor $(=-p \mathbf{I}+\mathbf{S}), p$ is the pressure, $\mathbf{I}$ is the identity tensor, $\mathbf{S}$ is the extra stress tensor $\left(=\frac{\mu}{1+\lambda_{1}}\left(\dot{\gamma}+\lambda_{2} \ddot{\gamma}\right), \dot{\gamma}=\nabla \mathbf{V}+(\nabla \mathbf{V})^{T}, \ddot{\gamma}=\frac{\mathrm{d}}{\mathrm{d}} \dot{\gamma}\right), \mu$ is the dynamic viscosity, $\lambda_{1}$ is the ratio of relaxation to retardation times, $\lambda_{2}$ is the retardation time and the dots over the quantities denote the material differentiation with respect to time, $F_{E}$ is the electrokinetic body force per unit volume. In electrokinetics, the fluid motion is induced by electric fields. The electric field is coupled with the fluid dynamics through the Lorentz's relation for the force on a charged species. The conservation equations for Jeffrey incompressible viscous aqueous fluid with $F_{E}=\rho_{e} E$ (where $\rho_{e}$ is the net charge density of the aqueous medium of permittivity $\varepsilon_{e}$ ), can be expressed as,

$$
\begin{gathered}
\frac{\partial u}{\partial x}+\frac{\partial v}{\partial y}=0 \\
\rho\left[\frac{\partial}{\partial t}+u \frac{\partial}{\partial x}+v \frac{\partial}{\partial y}\right] u=-\frac{\partial p}{\partial x}+\frac{\partial}{\partial x}\left(\tau_{x x}\right)+\frac{\partial}{\partial y}\left(\tau_{x y}\right)+\rho_{e} E \\
\rho\left[\frac{\partial}{\partial t}+u \frac{\partial}{\partial x}+v \frac{\partial}{\partial y}\right] v=-\frac{\partial p}{\partial y}+\frac{\partial}{\partial x}\left(\tau_{x y}\right)+\frac{\partial}{\partial y}\left(\tau_{y y}\right),
\end{gathered}
$$


where,

$$
\begin{aligned}
\tau_{x x} & =\frac{2 \mu}{1+\lambda_{1}}\left[1+\lambda_{2}\left(\frac{\partial}{\partial t}+u \frac{\partial}{\partial x}+v \frac{\partial}{\partial y}\right)\right] \frac{\partial u}{\partial x} \\
\tau_{x y} & =\frac{\mu}{1+\lambda_{1}}\left[1+\lambda_{2}\left(\frac{\partial}{\partial t}+u \frac{\partial}{\partial x}+v \frac{\partial}{\partial y}\right)\right]\left(\frac{\partial u}{\partial y}+\frac{\partial v}{\partial x}\right), \\
\tau_{y y} & =\frac{2 \mu}{1+\lambda_{1}}\left[1+\lambda_{2}\left(\frac{\partial}{\partial t}+u \frac{\partial}{\partial x}+v \frac{\partial}{\partial y}\right)\right] \frac{\partial v}{\partial y}
\end{aligned}
$$

The charge density $\rho_{e}$ can be related by Poissons equation of the electric potential of species $\Phi$, as $\rho_{e}=-\varepsilon_{e} \nabla^{2} \Phi$. The net charge density in a unit volume of the fluid, for binary and symmetric monovalent electrolyte containing two species (a monovalent cation with concentration $c_{+}$and a monovalent anion with concentration $c_{-}$with identical bulk concentration $\left.c_{0}\right)$, can be written as $\rho_{e}=-2 F c_{0} \sinh \left(\frac{F \Phi}{k_{B} T}\right)$, where, $F$ is the Faraday's constant, which is equal to the charge of one mole of ionic species ( $=e N_{A}, N_{A}$ is Avogadro number) and $T$ absolute temperature. The Poisson-Boltzmann equation for binary symmetric monovalent electrolyte solution can be simplified as

$$
\nabla^{2} \Phi=\frac{2 F c_{0}}{\varepsilon_{e}} \sinh \left(\frac{F \Phi}{k_{B} T}\right)
$$

This electric potential and ion distributions are de-coupled from the Navier-Stokes equations. Equation (5) can be linearized using the Debye-Hückel approximation. This approximation is valid for many bio-microfluidics applications, when an aqueous solution $\mathrm{pH}$ value is near neutral, the zeta potential value is around $-25 \mathrm{mV}$ or less (Kirby and Hasselbrink [31]).

When using the Debye-Hückel approximation $\left(\Phi<k_{B} T / e\right)$, equation (5) can be linearized as

$$
\nabla^{2} \Phi=\frac{2 c_{0} F^{2}}{\varepsilon_{e} k_{B} T} \Phi=\kappa^{2} \Phi
$$

where, $\xi=\sqrt{\frac{\varepsilon_{e} k_{B} T}{2 c_{0} F^{2}}}$ is the thickness of the Debye layer (Electric Double Later (EDL) thickness), associated with DebyeHückel parameter, $\kappa=\frac{1}{\xi}$.

\subsection{Lubrication theory}

The flow governing equations can be solved by employing lubrication approximation. It is assumed that Reynolds number $(R e)$, wall curvature $(\delta=a / \lambda)$ are very small (the local slope of the channel wall is small). The fluid flow pattern is 
described in non-dimensional form as

$$
\begin{aligned}
& x^{\prime}=\frac{x}{\lambda}, y^{\prime}=\frac{y}{a}, t^{\prime}=\frac{t}{T}, L^{\prime}=\frac{L}{\lambda}, \phi=\frac{b}{a}, h_{1}^{\prime}=\frac{h_{1}}{a}, h_{2}^{\prime}=\frac{h_{2}}{a}, u^{\prime}=\frac{u}{c}, \\
& v^{\prime}=\frac{v}{\delta c}, p^{\prime}=\frac{a^{2} p}{\mu c \lambda}, \Phi^{\prime}=\frac{\Phi}{\Phi_{0}}, \kappa^{\prime}=\kappa a, \operatorname{Re}=\frac{c a \delta}{v}, \tau^{\prime}=\frac{a \tau}{\mu c}, Q=\frac{\bar{Q}}{a c},
\end{aligned}
$$

where, $\delta$ defines wave number, $\phi$ is amplitude ratio/occlusion parameter and $\Phi_{0}=K_{B} T / e$ is the thermal potential. Moreover, substitution of equation (7) into mass and momentum equations (4) yields the dimensionless Stokes equations (after dropping primes) as

$$
\begin{aligned}
\frac{\partial u}{\partial x}+\frac{\partial v}{\partial y} & =0 \\
\operatorname{Re} \delta\left(\frac{\partial u}{\partial t}+u \frac{\partial u}{\partial x}+v \frac{\partial u}{\partial y}\right) & =-\frac{\partial p}{\partial x}+\delta \frac{\partial \tau_{x x}}{\partial x}+\frac{\partial \tau_{x y}}{\partial y}+U_{H S}\left(\delta^{2} \frac{\partial^{2} \Phi}{\partial x^{2}}+\frac{\partial^{2} \Phi}{\partial y^{2}}\right) \\
\operatorname{Re}^{3}\left(\frac{\partial v}{\partial t}+u \frac{\partial v}{\partial x}+v \frac{\partial v}{\partial y}\right) & =-\frac{\partial p}{\partial y}+\delta^{2} \frac{\partial \tau_{x y}}{\partial x}+\delta \frac{\partial \tau_{y y}}{\partial y}
\end{aligned}
$$

which are subject to the dimensionless boundary conditions

$$
\left.u\right|_{y=h_{1}}=\left.u\right|_{y=h_{2}}=0,\left.\quad v\right|_{y=h_{1}}=\frac{\partial h_{1}}{\partial t},\left.\quad v\right|_{y=h_{2}}=\frac{\partial h_{2}}{\partial t}
$$

where, $U_{H S}=-\frac{E \varepsilon_{e} \Phi_{0}}{\mu c}$ is called the ratio of the Helmholtz-Smoluchowski velocity to the characteristic wave velocity and $\Delta P_{L}(t)$ is the pressure difference between the channel ends.

The dimensionless form of equation (6), in $y$ direction can be written as

$$
\frac{\partial^{2} \Phi}{\partial y^{2}}=\kappa^{2} \Phi
$$

which may be solved subjected to $\Phi\left(h_{1}\right)=\zeta_{1}$ and $\Phi\left(h_{2}\right)=\zeta_{2}$ to yield

$$
\Phi(y)=\zeta_{1} \frac{\sinh \left[\kappa\left(y-h_{2}\right)\right]-R_{\zeta} \sinh \left[\kappa\left(y-h_{1}\right)\right]}{\sinh \left[\kappa\left(h_{1}-h_{2}\right)\right]}
$$

where, $R_{\zeta}=\zeta_{2} / \zeta_{1}$ is the ratio of zeta-potential of the two walls. Equation (11) is valid for $-h \leq y \leq h$ and the symmetric potential case of Bandopadhyay et al. [32] is obtained when $R_{\zeta}=1, \alpha=0, \theta=0$. 


\subsection{Rate of volume flux and boundary conditions}

The dimensional volume flow rate in laboratory frame is defined as

$$
\bar{Q}(x, t)=\int_{h_{1}}^{h_{2}} u(x, y, t) d y .
$$

The volume flow rate in laboratory frame can be expressed in the form of volume flow rate in wave frame as:

$$
\bar{Q}(x, t)=q(x)+c\left(h_{2}(x, t)-h_{1}(x, t)\right),
$$

and the time averaged flow rate over a period $T$, yielding

$$
Q_{T}(x)=q(x)-\phi+2(1+x \tan [\alpha])-\frac{\phi}{\pi} \frac{\cos [\pi(T-2 x)-\theta / 2] \cos [\theta / 2] \sin [\pi T]}{T} .
$$

It may be noted that for incompressible flow, prescribing volume flow rate is equivalent to prescribing normal velocity of the fluid at the wall. This is related via continuity equation,

$$
\frac{\partial \bar{Q}}{\partial x}(x, t)+\frac{\partial \bar{A}}{\partial t}(x, t)=0
$$

where $\bar{A}(x, t)$ is the cross sectional area. Integrating (15) with respect to $x$, we get

$$
\bar{Q}(x, t)=\bar{Q}(0, t)-\int_{0}^{x} \frac{\partial \bar{A}}{\partial t}(x, t) d x
$$

where $\bar{Q}(0, t)$ is the flow rate at $x=0$. However, due to equation of continuity, $Q_{T}(x)=Q_{T}$, for every $x$. Then the nondimensional form of volumetric flux can be obtained by substituting $q(x)$ from (14) in (13) and scaled with $a c$ as

$$
Q(x, t)=Q_{T}+\phi-2(1+x \tan [\alpha])+h_{2}(x, t)-h_{1}(x, t) .
$$


The stream function $\psi$ in dimensionless form can be defined as $u=\frac{\partial \psi}{\partial y}, v=-\frac{\partial \psi}{\partial x}$,

$$
Q(x, t)=\int_{h_{1}}^{h_{2}} \frac{\partial \psi}{\partial y} d y=\psi\left(h_{2}\right)-\psi\left(h_{1}\right)
$$

By the choice of $\psi\left(h_{1}\right)=-\frac{Q}{2}$ and $\psi\left(h_{2}\right)=\frac{Q}{2}$, utilizing lubrication theory, equations (8) and (9) after eliminating pressure term, can be written as

$$
\frac{\partial^{2}}{\partial y^{2}}\left[\frac{1}{1+\lambda_{1}} \frac{\partial^{2} \psi}{\partial y^{2}}\right]+U_{H S} \frac{\partial^{2} \Phi}{\partial y^{2}}=0
$$

subject to the dimensionless boundary conditions

$$
\psi\left(h_{1}\right)=-\frac{Q}{2}, \frac{\partial \psi}{\partial y}\left(h_{1}\right)=0, \quad \psi\left(h_{2}\right)=\frac{Q}{2}, \frac{\partial \psi}{\partial y}\left(h_{2}\right)=0
$$

\subsection{Analytical solutions}

The solution of equation (19) with the boundary conditions equations (20) is in the form:

$$
\begin{aligned}
\psi & =\frac{1}{2} \frac{\partial p}{\partial x}\left(h_{1} h_{2}-\frac{y}{2}\left(h_{1}+h_{2}\right)+\frac{y^{2}}{3}\right) y+\frac{Q\left(h_{1}+h_{2}\right)\left(h_{1}^{2}-4 h_{1} h_{2}+h_{2}^{2}\right)}{2\left(h_{1}-h_{2}\right)^{3}} \\
& +U_{H S}\left(1+\lambda_{1}\right) \zeta_{1}\left[I_{1}+R_{\zeta} I_{2}+\frac{R_{\zeta} \cosh \left[\kappa\left(y-h_{1}\right)-\cosh \left[\kappa\left(y-h_{2}\right)\right]\right.}{\kappa \sinh \left[\kappa\left(h_{1}-h_{2}\right)\right]}\right]
\end{aligned}
$$

where,

$$
\begin{gathered}
I_{1}=\frac{h_{2} y\left(2 h_{2}-y\right)+h_{1}\left(y^{2}-2 h_{2} y-2 h_{2}^{2}\right)}{2\left(h_{1}-h_{2}\right)^{2}}+\frac{h_{1}^{2}\left(h_{1}-3 h_{2}\right)+h_{2}^{2}\left(3 h_{1}-h_{2}\right) \cosh \left[\kappa\left(h_{1}-h_{2}\right)\right]}{\kappa \sinh \left[\kappa\left(h_{1}-h_{2}\right)\right]\left(h_{1}-h_{2}\right)^{3}}, \\
I_{2}=\frac{h_{2} y^{2}-2 h_{1}^{2}\left(h_{2}-y\right)-h_{1} y\left(2 h_{2}+y\right)}{2\left(h_{1}-h_{2}\right)^{2}}-\frac{h_{2}^{2}\left(3 h_{1}-h_{2}\right)+h_{1}^{2}\left(h_{1}-3 h_{2}\right) \cosh \left[\kappa\left(h_{1}-h_{2}\right)\right]}{\kappa \sinh \left[\kappa\left(h_{1}-h_{2}\right)\right]\left(h_{1}-h_{2}\right)^{3}} .
\end{gathered}
$$

The axial velocity can be obtained from the equation (21) as

$$
u=\frac{1}{2} \frac{\partial p}{\partial x}\left(y-h_{1}\right)\left(y-h_{2}\right)-U_{H S}\left(1+\lambda_{1}\right) \zeta_{1}\left[\frac{R_{\zeta}\left(y-h_{1}\right)}{h_{1}-h_{2}}-\frac{\left(y-h_{2}\right)}{h_{1}-h_{2}}+\frac{\Phi}{\zeta_{1}}\right] .
$$


Table 1. Typical physical and electrochemical parameters.

\begin{tabular}{ccc}
\hline \hline Parameter & Symbol & Range [32-35] \\
\hline Typical channel height $(\mu \mathrm{m})$ & $2 a$ & $10 \sim 5000$ \\
Electrolyte concentration $(\mathrm{mM})$ & $c_{0}$ & $0.001 \sim 10$ \\
Debye length $(\mathrm{nm})$ & $\xi$ & $1 \sim 100$ \\
Zeta potential $(\mathrm{mV})$ & $\zeta$ & $\pm 1 \sim \pm 100$ \\
Electric field $(\mathrm{kV} / \mathrm{cm})$ & $E$ & $\sim 1$ \\
Electroosmotic Velocity $(\mathrm{cm} / \mathrm{s})$ & $U_{H S}$ & $\sim 2$ \\
\hline \hline
\end{tabular}

The derivation for the present model is similar to the symmetric Newtonian fluid model of Bandopadhyay et al. [32]. Moreover, the expression of the axial pressure gradient can be obtained from equations (8), (16) and (22) as

$$
\frac{\partial p}{\partial x}=\frac{f(t)+2 \int_{0}^{x}\left(\frac{\partial h_{2}(s, t)}{\partial t}-\frac{\partial h_{1}(s, t)}{\partial t}\right) d s}{\frac{1}{6}\left(h_{2}^{3}-h_{1}^{3}\right)+\frac{h_{1} h_{2}}{2}\left(h_{1}-h_{2}\right)}+\frac{U_{H S} \zeta_{1}\left(1+\lambda_{1}\right)\left(1+R_{\zeta}\right)\left[\left(h_{2}-h_{1}\right)+\frac{2}{\kappa} \tanh \left[\frac{\kappa\left(h_{1}-h_{2}\right)}{2}\right]\right]}{\frac{1}{6}\left(h_{2}^{3}-h_{1}^{3}\right)+\frac{h_{1} h_{2}}{2}\left(h_{1}-h_{2}\right)},
$$

The arbitrary function $f(t)$ is determined by integration of equation (23) over the finite length of the channel $(x=0$ to $x=L)$

$$
f(t)=\frac{\Delta P_{L}(t)-12 \int_{0}^{L} \frac{0}{\int_{2}^{x}\left(\frac{\partial h_{2}(s, t)}{\partial t}-\frac{\partial h_{1}(s, t)}{\partial t}\right) d s} d x \int_{0}^{L} \frac{\int_{0}^{2} \frac{U_{H S} \zeta_{1}\left(1+\lambda_{1}\right)\left(1+R_{\zeta}\right)\left[\left(h_{2}-h_{1}\right)+\frac{2}{\kappa} \tanh \left[\frac{\kappa\left(h_{1}-h_{2}\right)}{2}\right]\right]}{\left(h_{1}\left(h_{1}-h_{2}\right)\right.} d x}{\left(h_{2}^{3}-h_{1}^{3}\right)+3 h_{1} h_{2}\left(h_{1}-h_{2}\right)} d x}{\int_{0}^{L} \frac{d x}{\left(h_{2}^{3}-h_{1}^{3}\right)+3 h_{1} h_{2}\left(h_{1}-h_{2}\right)}} .
$$

From the equations (13) and (14), the axial pressure gradient in terms of time-averaged flow can be presented in the form:

$$
\frac{\partial p}{\partial x}=\frac{2 Q(x, t)+U_{H S} \zeta_{1}\left(1+\lambda_{1}\right)\left(1+R_{\zeta}\right)\left[\left(h_{1}-h_{2}\right)-\frac{2}{\kappa} \tanh \left[\frac{\kappa\left(h_{1}-h_{2}\right)}{2}\right]\right]}{\frac{1}{6}\left(h_{1}^{3}-h_{2}^{3}\right)+\frac{h_{1} h_{2}}{2}\left(h_{2}-h_{1}\right)},
$$

The local wall shear stress derived at lower and upper walls are respectively

$$
\begin{aligned}
& \tau_{w_{1}}(x, t)=\frac{1}{2} \frac{\partial p}{\partial x}\left(h_{1}-h_{2}\right)+U_{H S} \zeta_{1}\left(1+\lambda_{1}\right)\left[\frac{1-R_{\zeta}}{h_{1}-h_{2}}-\frac{\Phi^{\prime}\left(h_{1}\right)}{\zeta_{1}}\right] \\
& \tau_{w_{2}}(x, t)=\frac{1}{2} \frac{\partial p}{\partial x}\left(h_{2}-h_{1}\right)+U_{H S} \zeta_{1}\left(1+\lambda_{1}\right)\left[\frac{1-R_{\zeta}}{h_{1}-h_{2}}-\frac{\Phi^{\prime}\left(h_{2}\right)}{\zeta_{1}}\right] .
\end{aligned}
$$




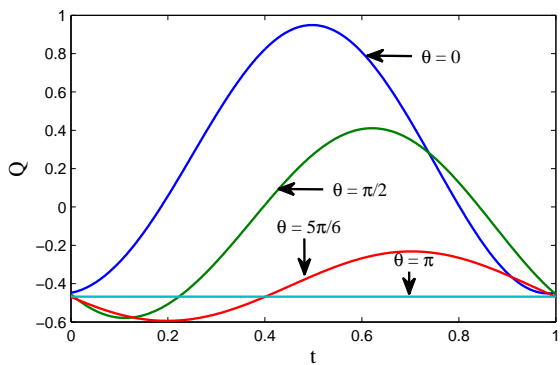

(a)

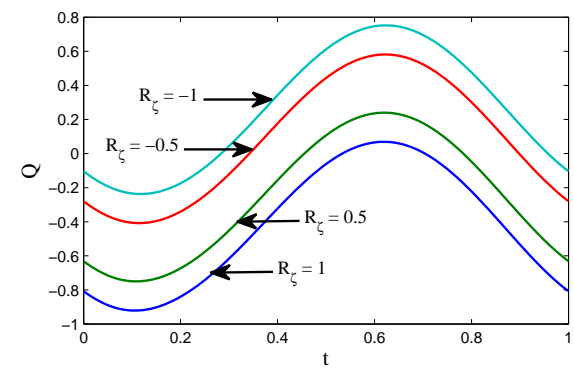

(b)

Fig. 2. Instantaneous flow rates for (a) various phase shifts $\theta$ when $R_{\zeta}=-0.5$, and (b) zeta-potential ratios $R_{\zeta}$ during a single period when $\theta=\pi / 2\left(L=1, \phi=0.7, \lambda_{1}=1, \alpha=0.035, U_{H S}=-1, \kappa=5\right)$.

\section{Numerical results and discussion}

Intrauterine transport driven by myometrial contractions was simulated for an artificial uterus geometry. In Sect. 2.4, the solution was obtained for unsteady peristaltic flow of viscoelastic fluid (Jeffrey model) under the mixed influence of pressure gradient due to oscillating walls $\left(p_{0}=p_{L}=0\right)$ and electro-osmosis induced by asymmetric zeta potentials at the tapered channel walls. The average values for the model were: $2 a=1 \mathrm{~mm}, L=100 a, \lambda=50 a, \phi=0.7$, and $\alpha=0.04 \mathrm{rad}$. Typical physical and electrochemical parameter ranges employed in electrokinetic flows are given in Table 1.

The flow rate of the uterus model was analyzed for different phase shifts $\theta(0, \pi / 2,5 \pi / 6, \pi)$ between the channel walls during single period in Fig. 2(a). The analysis is presented for three different cases of Helmholtz-Smoluchowski electroosmotic velocity $U_{H S}$, each illustrating the case of aiding, opposing and no applied electric field. The aiding electric field can be obtained when $U_{H S}$ and $R_{\zeta}$ have both positive or negative values. The opposing electric field can be archived when $U_{H S}$ and $R_{\zeta}$ have opposite signs and there is no electric field when $U_{H S}=0$. The maximal flow rate is observed for symmetric wall motility $(\theta=0)$ and decreases as phase shift between the channel walls approaches to out of phase value $(\theta \rightarrow \pi)$. Flow rates are greatest with symmetric wall motility $(\theta=0)$, for the channel walls have synchronized movement as they act in phase and propelling the fluid forward. Moreover, as the walls move in asymmetric manner $(\theta \rightarrow \pi)$, the wall movement contributes different forces from both sides of the channel at any spacial and temporal point, with consequent decline in total flow rate. Figure 2(b) illustrates the variation of flow rate for different values of zeta potential ratios $R_{\zeta}(-1,-0.5,0.5,1)$. The volumetric flow rate is a decreasing function of $R_{\zeta}$. In other words, the flow rate enhances with the ratio of zeta potentials decreases from symmetric $\left(R_{\zeta}=1\right)$ to anti-symmetric $\left(R_{\zeta}=-1\right)$. The dependency of the time averaged flow rate on the length of the channel is depicted in Fig. 3(a) for uniform $(\alpha=0)$ and non-uniform $(\alpha=0.1,0.2,0.3)$ channel for anti-symmetric zeta potentials $R_{\zeta}=0.5$. The curves are in a slight oscillatory nature and are periodic with wave length. The magnitude of oscillation is a decreasing function of length of the channel. The average slope of curves is seen to decrease in non-uniform channels as the tapered angle of the channel increases. The variability of the net flow rate $\left(Q_{T}\right)$ with various phase shifts $(\theta)$ for different values of zeta-potential ratios $\left(R_{\zeta}\right)$ is depicted in Fig. 3(b). It is clearly shown that the net flow rate decreases as wall motility tends to extremely asymmetric (i.e. $\theta \rightarrow \pi$ ), but it increases as zeta-potential ratio $\left(R_{\zeta}\right)$ varying from symmetric $\left(R_{\zeta}=1\right)$ to anti-symmetric $\left(R_{\zeta}=-1\right)$. It is also evident from the figure that the peristaltic transport cannot 


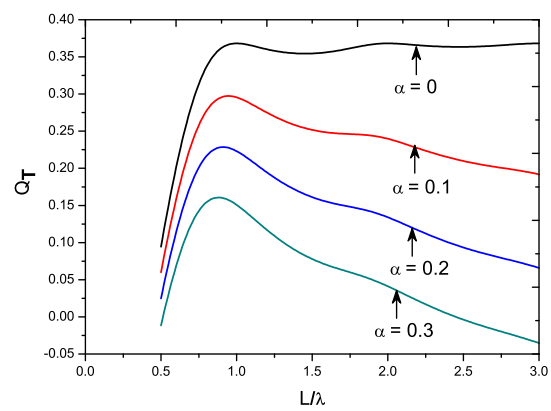

(a)

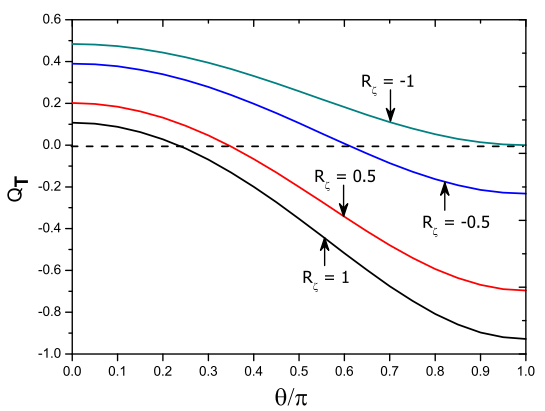

(b)

Fig. 3. The total flow rate over one period for (a) angle of inclination between the channel walls $\alpha$ when $R_{\zeta}=0.5$, and (b) zeta-potential ratios when $\alpha=0.035$.

act as a pump ( $Q_{T}<0$, i.e. peristalsis is completely eliminated) at large values of $\theta$ and at decreased values of zeta potential ratios.
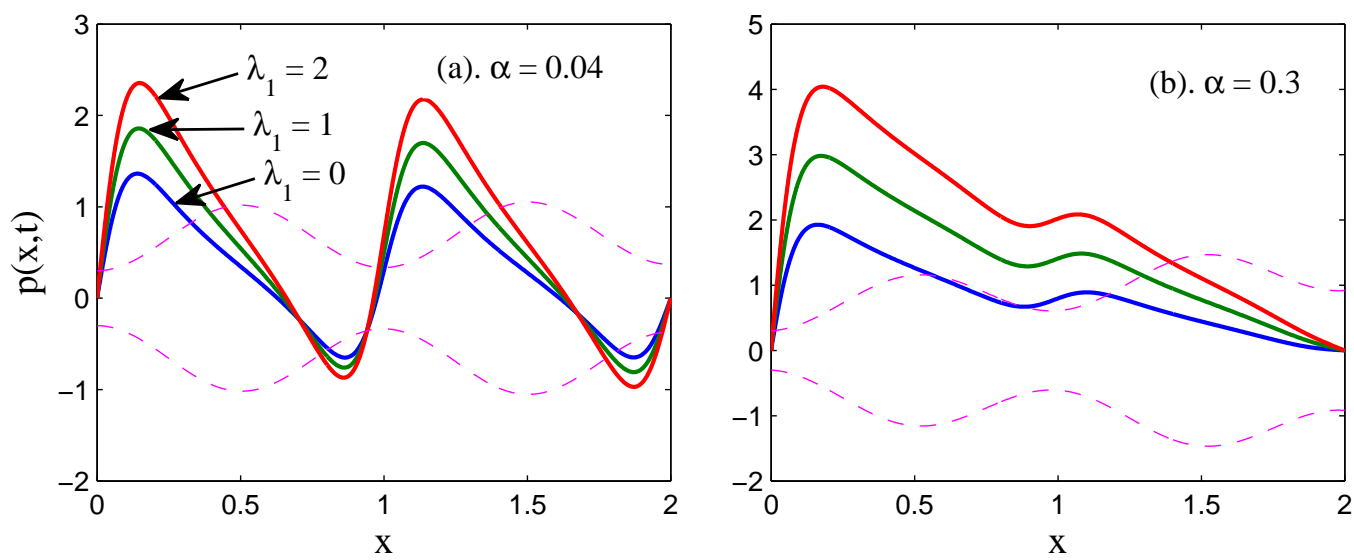

Fig. 4. Local pressure distribution along the channel axis for different inclination angle $\alpha$ and viscoelastic parameter $\left(\lambda_{1}\right)$ with $L=2, \phi=$ $0.7, \theta=0, U_{H S}=-1, \kappa=2, R_{\zeta}=0.5$.
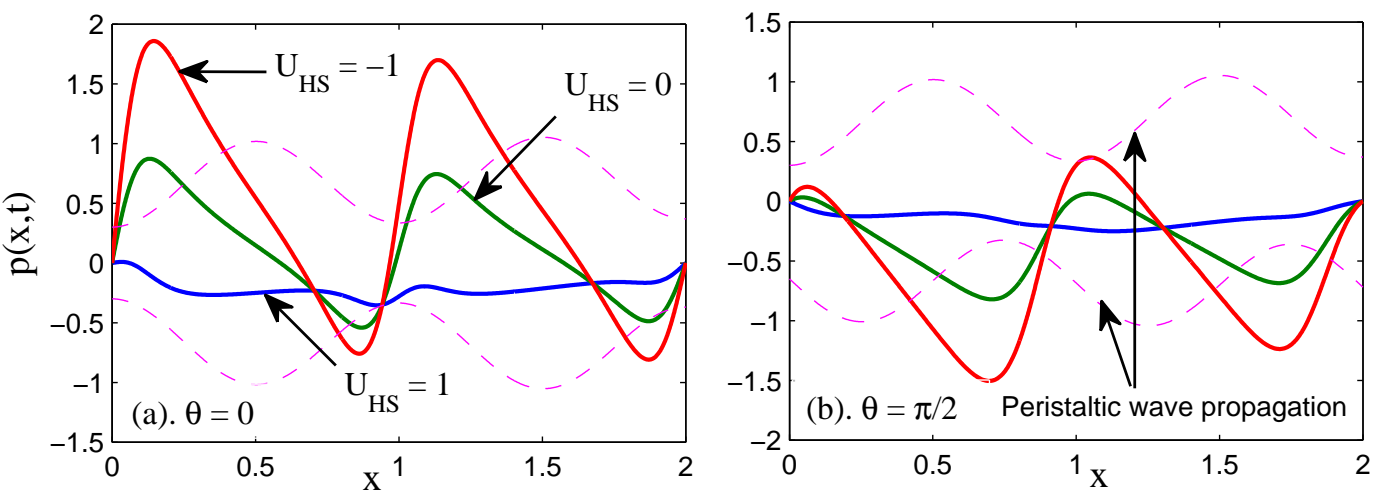

Fig. 5. Local pressure distribution along the channel axis for various phase shifts $(\theta)$ and electroosmotic velocity $\left(U_{H S}\right)$ with $L=2, \phi=$ $0.7, \alpha=0.04, \lambda_{1}=1, \kappa=2, R_{\zeta}=0.5$. 
Figure 4 illustrates the distribution of local pressure with different inclination angle $(\alpha=0.04,0.3)$ for various values of viscoelastic parameter $\left(\lambda_{1}\right)$. The case of a Newtonian fluid is retrieved when relaxation and retardation times are neglected in the Jeffrey model $\left(\lambda_{1}=0\right)$ and this achieves lower pressure. As expected, increasing viscoelastic parameter $\left(\lambda_{1}\right)$ increases the pressure amplitudes. It is also observed from this figure that the pressure rises sharply at the inlet, reaches maximum magnitude, then falls to zero at the middle of the bolus, comes down further to minimum magnitude and finally rises eventually to meet the leading end of the bolus. The same sinusoidal profile is repeated for the next bolus with huge control in the case of uniform channel $(\alpha=0)$. The periodicity of the pressure distribution is lost, when there is non-uniformity in the channel $(\alpha>0)$. Larger inclination angles between the walls result in higher pressure amplitudes at the inlet of the channel. The pressure distribution along the axis of the tapered channel decreases towards the wide exit of the channel (Fig. 4(b)). The dependency of the axial pressure along the flow passage length for different electroosmotic velocity $\left(U_{H S}\right)$, and phase shift $(\theta)$ is depicted in Fig. 5(a and b). The amplitudes of pressure profile decrease while moving from symmetric channel $(\theta=0)$ to asymmetric channel $(\theta \rightarrow \infty)$. The magnitude of pressure increases by negative values of $U_{H S}$ and decreases by positive values of $U_{H S}$ at the positive zeta-potential boundary values. This is perhaps due to the fact that negative HelmholtzSmoluchowski velocity $\left(U_{H S}<0\right)$ opposes the peristaltic flow direction and this leads to a maximum pressure amplitude throughout.
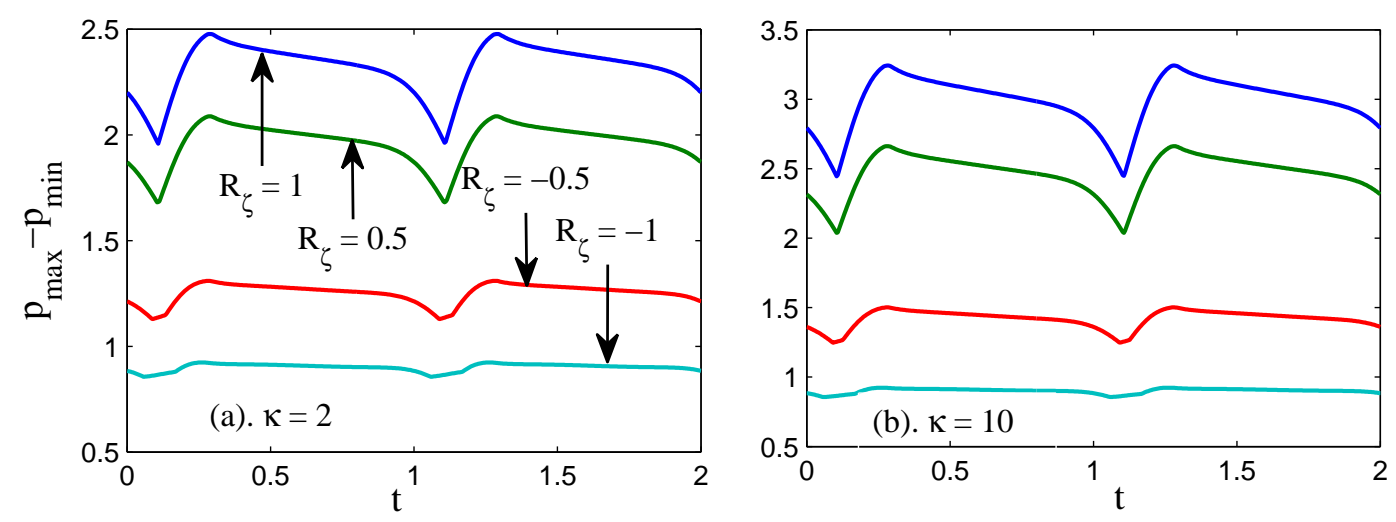

Fig. 6. Maximum-to-minimum pressure differences in a non-uniform channel during two periods for various zeta potential ratios $\left(R_{\zeta}\right)$ and Debye-Hückel parameter ( $\kappa$ ) with $L=2, \phi=0.7, \alpha=0.04, \lambda_{1}=1, \theta=\pi / 2, \Delta P_{L}=0, U_{H S}=-1$.

The maximum-to-minimum pressure differences of local pressure distribution in a finite non-uniform channel $(\alpha=0.04)$ for different values of zeta-potential ratio and electro-osmotic parameter (inverse Debye length) with time are shown in Fig. 6. The magnitude of maximum-to-minimum pressure differences is an increasing function of inverse Debye length ( $\kappa)$. As the $R_{\zeta}$ increases from -1 to 1 with $U_{H S}<0$, the pressure and maximum-to-minimum differences increase. When $U_{H S}>0$, the pressure and maximum-to-minimum differences decrease.

Figure 7 depicts the influence of zeta-potential ratio on local wall shear stress along the length of non-uniform channel. The observation of shear stress in the considered model is significant, because the amount of shear stress is proportional to the net amount of power needed to push fluid through the channel. Equation (26) clearly indicates the influence of 

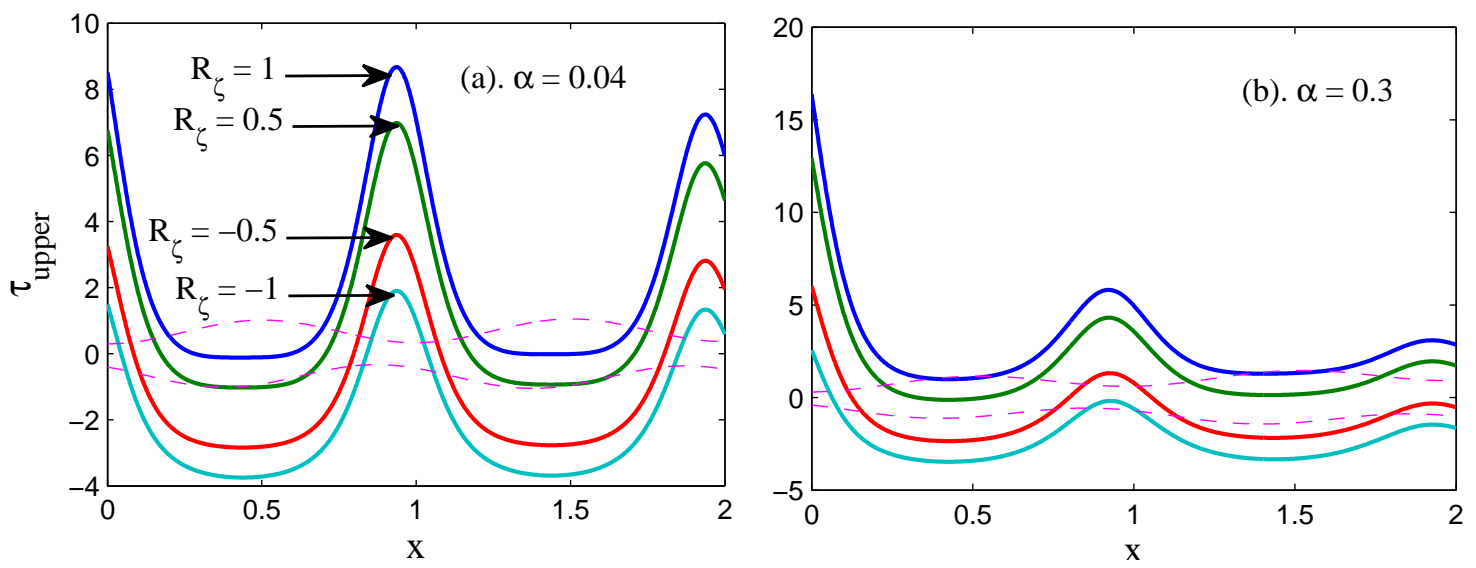

Fig. 7. Spatial variation of the wall shear stress for various zeta potential rations $\left(R_{\zeta}\right)$ when there are different inclination angles $(\alpha)$ between the walls with $L=2, \phi=0.7, \Delta P_{L}=0, \theta=\pi / 4, \lambda_{1}=1, U_{H S}=-1, \kappa=2$.

the electrokinetic body force towards the non-dimensional wall shear stress. This equation is valid for symmetric channel $(-h \leq y \leq h)$ and, $\tau_{w_{2}}$ when $h_{1}=-h_{2}=h, R_{\zeta}=1$, and $\zeta_{1}=\zeta$, the symmetric wall shear stress $\tau_{w}$ is recovered (Bandopadhyay et al. [32]). It is observed that wall shear stress is positive in the region of channel occlusion. The shear stress distribution increases with positive $R_{\zeta}$ and decreases with negative $R_{\zeta}$ when $U_{H S}=-1$. This indicates that the magnitude of the wall shear stress increases where the electric field opposes the net flow direction. As the inclination angle between the walls increases the shear stress values increase at inlet and decay rapidly towards the wide exit of the tapered channel. It is also noted that the behavior of the shear stress at lower wall $\tau_{w_{1}}$ is opposite to that of the shear stress at upper wall $\tau_{w_{2}}$ under the influence of all parameters.

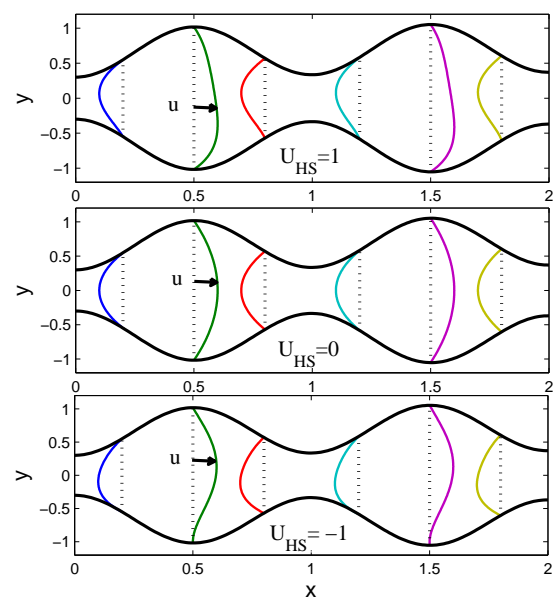

(a) $\alpha=0.04$

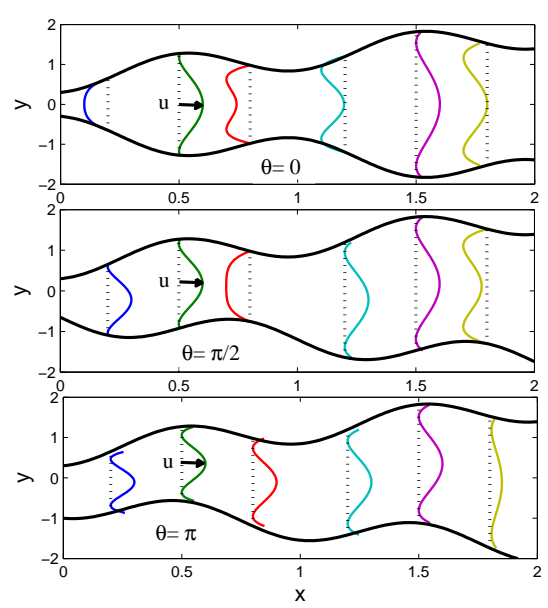

(b) $\alpha=0.5$

Fig. 8. Axial velocity for different $(a) U_{H S}(1,0,-1)$ and $(b) \theta(0, \pi / 2, \pi)$ when $\phi=0.7, \lambda_{1}=1, R_{\zeta}=1, \kappa=2, Q_{T} / Q_{T, \operatorname{Max}}=0.01$.

The velocity distributions at different cross sections along the channel passage length at a given time are presented in Fig. 8. The fluid flow is induced by the combined effect of peristalsis and external electric field that alternates forward and backward motion. The forward motion occurs in wider cross sectional regions of the channel. In the narrow cross sectional 
regions, the flow alters direction opposite to the peristaltic wave propagation direction. The profiles are similar to those in a model with no force of applied electric field [5,9]. The peristaltic motion can be controlled by electro-osmosis. Figure 8(a) illustrates the effects of Helmholtz-Smoluchowski velocity $\left(U_{H S}\right)$ on fluid velocity in a channel with inclination angle $\alpha=0.04$. For $U_{H S}=1$, the maximal velocity is shifted towards lower (inner) half-space at wider cross sectional region where forward motion occurs. The opposite effect is manifested with backward motion where the profile resides at narrow cross sectional region. The velocity distribution is therefore asymmetric across the channel. The velocity profile with no applied electric field $\left(U_{H S}=0\right)$ corresponds is a parabolic curve across the channel. For $U_{H S}=-1$, it is observed that the behavior of the velocity curve is opposite to that of the velocity curve for $U_{H S}=1$. The influence of phase shift on axial velocity in a tapered channel is depicted in Fig. 8(b). It is observed from the figure that the backward motion is greatest when there is symmetric wall propagation $(\theta=0)$ since both the peristaltic walls act in phase with huge control. When $\theta \rightarrow \pi$, the wall movements are not in synchronized manner and electro-osmotic force dominates peristalsis and alters velocity profiles in forward direction eventually. As the channel walls propagate a "snake like" pattern $(\theta=0)$, there is still axial motion due to electro-osmosis. If there is no external electric force the results are in agreement with zero axial velocities from the previous study of Eytan et al. [9].

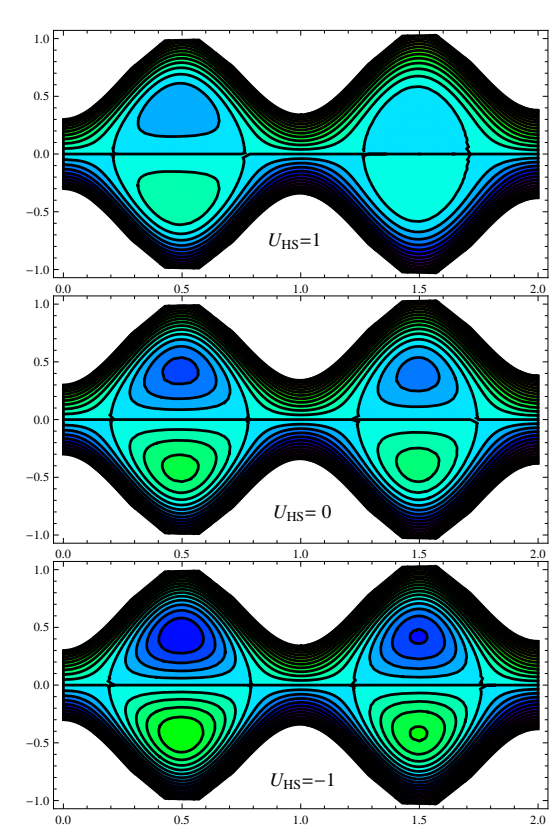

(a) $\alpha=0.04$

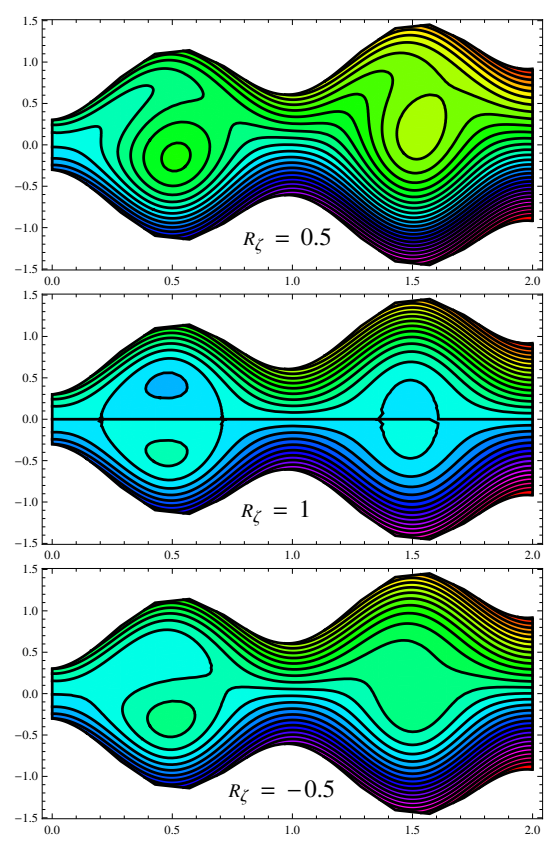

(b) $\alpha=0.5$

Fig. 9. Stream line patterns for different $(a) U_{H S}(1,0,-1)$ and $(b) R_{\zeta}(0.5,1,-0.5)$ when $\phi=0.7, \theta=0, \lambda_{1}=1, \kappa=2, Q_{T}=0.95$.

Trapping is an interesting phenomenon in peristaltic transport. The stream line in wave frame under certain conditions (with stagnation points) split to trap a fluid bolus which propagates along the channel with the wave speed. The effect of inclination angle $(\alpha)$ on trapping is shown in Fig. 9. It should be noted that the size of the trapped bolus decreases as the passive cross section increases. Figure 9(a) visualize the stream line patterns in the channel for different values of electro- 
osmotic velocity $\left(U_{H S}\right)$. A weak fluid trapping is noticed near the central line of the channel in symmetric manner for the case electro-osmosis opposes slightly the peristalsis $\left(U_{H S}=1\right)$, while there is a strong trapping near the centerline of the channel for the case where electro-osmosis aids the peristalsis $\left(U_{H S}=-1\right)$. It is clear that the opposing peristaltic motion leads to weaker bolus growth while in the case of an aiding peristaltic motion, a much stronger bolus growth is witnessed in the two symmetric boluses near the central line of the channel. Figure 9(b) depicted the trapping for various zeta-potential ratio $\left(R_{\zeta}\right)$. For symmetric boundary conditions $\left(R_{\zeta}=1\right)$, The fluid particles get trapped in a bolus symmetrically near the centerline and then continuous to decrease. The size of the particle trapping depends on its location along the channel length. For the case of asymmetric boundary conditions $\left(R_{\zeta}=0.5,-0.5\right)$, trapping is noticed near the upper wall of the channel and the size of the trapping is found to be correlated with the plot of maximum-to-minimum pressure difference (Fig. 6).

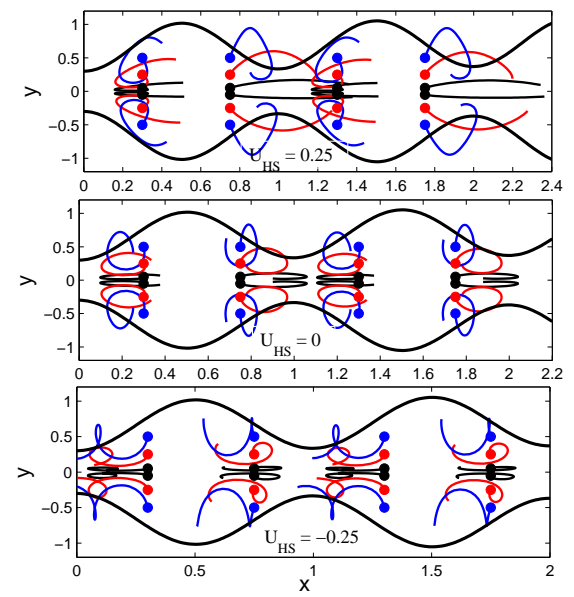

(a) $\alpha=0.04$

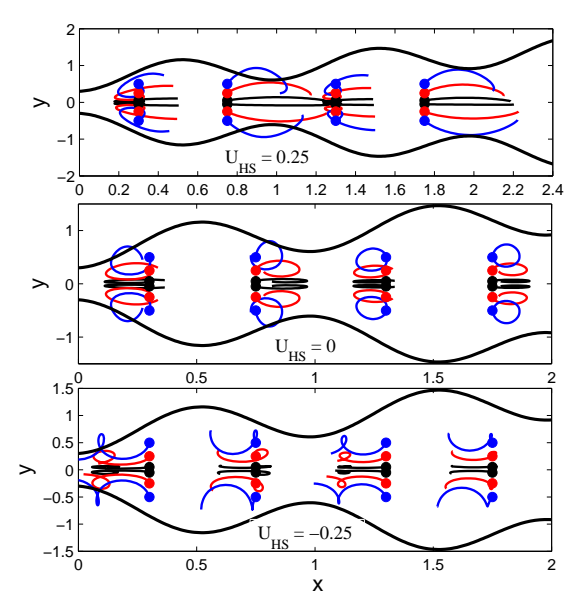

(b) $\alpha=0.3$

Fig. 10. Particles trajectories during one period $(T)$ when there are different $(a) U_{H S}(0.25,0,-0.25)$ and $(b) \alpha(0.04,0.3)$ with $\phi=$ $0.7, \lambda_{1}=1, R_{\zeta}=0.5, \kappa=2, Q_{T} / Q_{T, \operatorname{Max}}=0.01$.

Reflux or retrograde motion is another interesting phenomenon in peristaltic transport which can be computed by particle advection process in the Lagrangian point of view during the time the peristaltic wave completes one full cycle. Such Lagrangian trajectory of the massless fluid particle can be modeled by velocity field $\mathbf{V}(\mathbf{X}, \mathbf{t}) \in \mathbb{R}^{n}$. Consider the initial position of the massless particle is $\mathbf{X}_{\mathbf{0}}$ at time $t=t_{0}$, then the resulting particle advection is specified by the initial value problem (IVP) $\dot{\mathbf{X}}=\mathbf{V}(\mathbf{X}, \mathbf{t}), \mathbf{X} \in \mathbb{R}^{n}, t \geq t_{0}$ subject to $\mathbf{X}=\mathbf{X}_{0}$ at $t=t_{0}$. The fluid particle trajectory may be computed by simultaneous integration of these advection equations using a numerical ODE integrator, such as Runge-Kutta method. Figure 10 shows the trajectories of six fluid particles initially located at several cross sections along the channel for different electro-osmosis velocities $\left(U_{H S}\right)$ and two different inclination angles $(\alpha=0.04,0.3)$. The net axial displacement of the particles near the walls increase with inclined angle and the particles net axial displacement near the centerline decrease with inclined angle. Figures 10(a) and 10(b) demonstrate that retrograde motion is most likely to start near the channel walls in the absence of applied electric field (i.e. $U_{H S}=0$ ) as indicated by previous studies [9,36]. A positive value of $U_{H S}(=0.25)$ implies an added axial flow and hence an increase in the net axial displacement of massless particles. However, in the case 


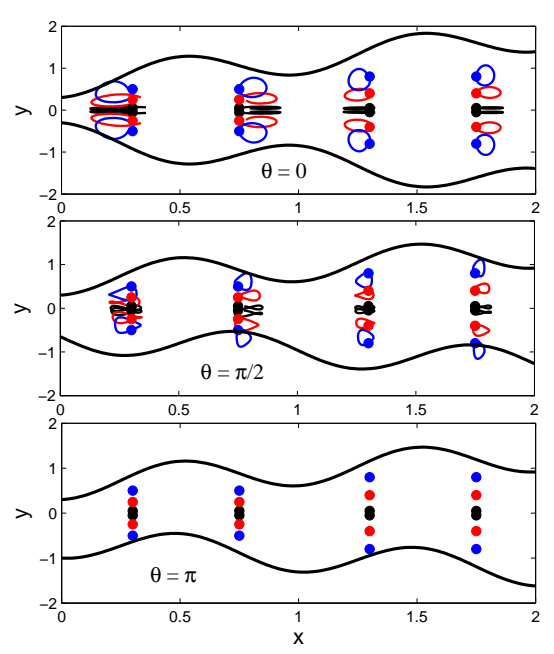

(a) $U_{H S}=0$

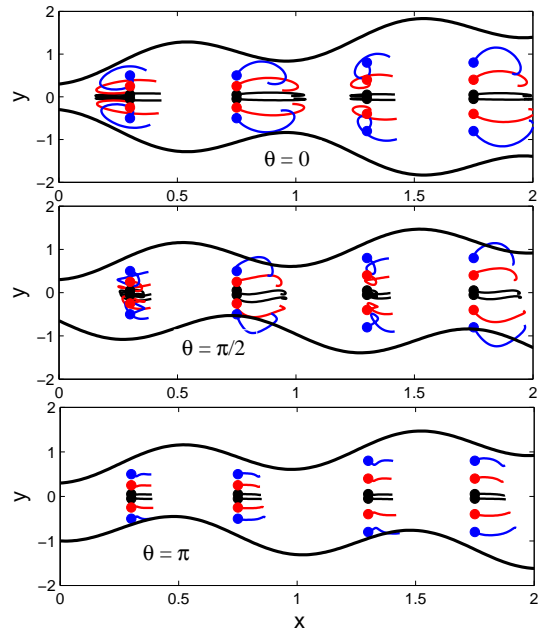

(b) $U_{H S}=0.25$

Fig. 11. Particles trajectories during one period $(T)$ when there are various phase shifts $(\theta)$ when $\phi=0.7, \alpha=0.5, \lambda_{1}=0, R_{\zeta}=0.5, \kappa=$ 2, $Q_{T} / Q_{T, \text { Max }}=0.01$.

of negative value of $U_{H S}(=-0.25)$, the electro-osmotic flow opposing the axial flow. It is observed that massless particles in the channel undergo a net negative displacement, leading to retrograde motion (Reflux). Path lines traced in Fig. 11 for wall propagation with various phase shifts $(\alpha=0, \pi / 2, \pi)$ are under the influence of electrical body force. It is already observed in the previous figures that an applied electric body force enhances the particle mobility in axial direction. The peristaltic force on a fluid particle is greatest when there is symmetric wall motility $(\theta=0)$, which leads to maximal net axial displacement of particles. There is a decline in the particle mobility in axial direction when the walls are propagated asymmetrically. Since both walls act with an unsynchronized motility (due to some phase difference), it leads to eventual decrease in net axial displacement of particles. It is also observed from this figure that the axial transport of the particles is zero at $U_{H S}=0$ and $\theta=0$ as shown in the previous literature [5,9]. There is still significant particle mobility in axial direction at $\theta=0$ under an applied electric body force as shown in Fig. 11(b). Therefore electro-osmosis play a significant role to transport embryo by the uterine fluid for successful reproduction.

\section{Microfluidics application to uterine peristalsis}

A uterus generally has a closed cavity at the fundus end. A small pressure gradient may develop when embryo hits the closed fundus wall. This pressure gradient may initiate a retrograde motion which may act as a mechanical factor of infertility. This may also shift the embryo away from the fundus. Thus, irregular uterine motility, uterine reflux, hydrosalpinx (i.e. a distally blocked fallopian tube filled with serous or clear fluid) may introduce problems of infertility. In in vivo successful reproduction, the embryo (a ball of cells about $100-150 \mu \mathrm{m}$ ) takes $3-4$ days to be carried from the fallopian tube to the fundus area (the upper part of the uterine cavity) by the intrauterine fluid motion. The phenomenon involves biochemical, hormonal and mechanical aspects. On the other hand, the embryo is transferred via a thin catheter using in vitro fertilization (IVF) in the laboratory. The successful fertilization of ova in the laboratory is obtained in $90 \%$ of the cases, whereas successful birth rates are improved only in $20-25 \%$. The poor birth rates may be explained by the failure to 
implant the embryo. Understanding of the dynamics of embryo transport to a successful implantation may open new doors for the development of effective strategies to enhance the embryo transfer rate during in vitro fertilization (IVF) which is meant for aiding women with fertility problems (Yaniv et al. [3,37]). It is noteworthy from the existing literature that higher magnitudes of intrauterine pressure yields higher stresses within the uterine wall. This represents hyperperistalsis and lead to endometrial tissue damage and sprout the development of pathologies. [38]. Cervix expands in diameter from $1 \mathrm{~cm}$ to 10 and ligaments also remodel during this course. This alters the mechanical behavior of the uterus. Moreover, modifications in mechanical behavior may also occur with age or due to hormonal changes and number of pregnancies. These alterations may lead to different wombs in the same women during her lifespan (Payan et al. [39] ).

Any change in mechanical properties of the uterus will influence the pattern of peristalsis-induced intrauterine fluid motion. These changes may ultimately affect the implantation and childbirth. Thus, the regulation of intrauterine fluid is important to plan intervention strategies. The present study explains how an external stimulus e.g. electric and magnetic fields influence the intrauterine fluid motion. The model presented in this work also helps us to understand the effect of electric field on intrauterine fluid flow. The findings of the present model will be helpful in improving the simulation studies of intrauterine fluid flow induced by peristaltic pumping and its behavior under the influence of electrohydrodynamics. Engineers may use this formulation to evaluate the performance of the microfluidic device designed for the regulation of intrauterine fluid motion. An advanced bio microfluidics device can also be developed which can implant the embryo at a suitable place in the uterus. Based on the principle discussed in the present study, effective strategies may also be planned to prevent pre-term delivery. Ultimately, this work contributes to the area of fertility research and human health care.

The results of the present model raise several points of interest related to aiding electrical force enhances the particle axial motility. The maximum intrauterine fluid velocities, flow rates and overall transport of massless particles were achieved when there was small phase shift $(\phi \rightarrow 0)$, inclination angle tended to zero $(\theta \rightarrow 0)$ and the electric field implied an added axial flow.

\section{Conclusions}

A mathematical model is considered to simulate the peristaltic transport of a viscoelastic fluid (Jeffrey model) in a tapered $2 D$ channel under the influence of electro-osmosis induced by asymmetric zeta potentials at the channel walls. This model explains significant physiological relevance with an ever increasing desire to study intrauterine fluid flow motion in a non-pregnant uterus. The consequences of this model may introduce a new mechanical factor for embryo transport to a successful implantation site at the upper part of the uterine cavity (fundus area). Based on the results, the following conclusions can be noted:

1. The transport phenomena are strongly affected by electro-osmotic force.

2. Total flow rates and axial velocities are greatest when there is an electro-osmotic force aiding the axial flow with the choice of zeta potential ratio.

3. The trapped bolus size is correlated with changing parameter values of maximum-to-minimum pressure difference. 
4. Strong fluid trapping is noticed near the central line of the channel for the case where electro-osmosis opposes axil flow.

5. The size of the trapped bolus is a decreasing function of tapered channel length.

6. Electro-osmotic force enhances the particle axial motility even though the walls are out of phase.

\section{References}

[1] R. J. Lang, M. E. Davidson, B. Exintaris, Pyeloureteral motility and ureteral peristalsis: essential role of sensory nerves and endogenous prostaglandins, Exp Physiol. 87(2) (2002) 129-146.

[2] M. Ezzati, O. Djahanbakhch, S. Arian, B. R. Carr, Tubal transport of gametes and embryos: a review of physiology and pathophysiology, Journal of Assisted Reproduction and Genetics 31 (2014) 1337-1347.

[3] S. Yaniv, D. Elad, A. J. Jaffa, O. Eytan, Biofluid aspects of embryo transfer, Ann. Biomed. Eng. 31 (2003) 1255.

[4] C. Bulletti, D. D. Ziegler, Uterine contractility and embryo implantation, Curr. Opin. Obstet. Gynecol. 17 (2005) $265-$ 276.

[5] O. Eytan, D. Elad, Analysis of intra-uterine fluid motion induced by uterine contractions, Bull. Math. Biol. 61(2) (1999) $221-238$.

[6] L. Zhu, Y. Li, A. Xu, Influence of controlled ovarian hyper-stimulation on uterine peristalsis in infertile women, Hum Reproduction 27 (2012) 2684-2689.

[7] L. Zhu, H. S. Che, L. Xiao, Y. P. Li, Uterine peristalsis before embryo transfer affects the chance of clinical pregnancy in fresh and frozen-thawed embryo transfer cycles, Human Reproduction 29 (2014) 1238-1243.

[8] V. Aranda, R. Cortez, L. Fauci, A model of stokesian peristalsis and vesicle transport in a three-dimensional closed cavity, Urol Int. 37(4) (2015) 240-246.

[9] O. Eytan, A. J. Jaffa, D. Elad, Peristaltic flow in a tapered channel: application to embryo transport within the uterine cavity, Medical engineering \& physics 23(7) (2001) 475-484.

[10] O. Eytan, D. Elad, U. Zaretsky, A. J. Jaffa, A glance into the uterus during in vitro simulation of embryo transfer, Hum. Reprod. 19 (2004) 562-569.

[11] S. Yao (Ed.), Electro-Osmotic Pump Technologies: Theory, Design and Demonstration.

[12] J. Dainty, P. C. Croghan, D. S. Fensom, Electro-osmosis, with some applications to plant physiology, Canadian Journal of Botany 41(6) (1963) 953-966.

[13] A. J. Kalmijn, The electric sense of sharks and rays, Exp. Biol. 55 (1971) 371-383.

[14] T. Tandon, B. J. Kirby, Zeta potential and electroosmotic mobility in microfluidic devices fabricated from hydrophobic polymers: 2. slip and interfacial water structure, Electrophoresis 29(5) (2008) 1102-1114.

[15] M. Brown, C. Meinhart, Ac electroosmotic flow in a dna concentrator, Microfluidics and Nanofluidics 2 (2006) 513523.

[16] D. Tripathi, S. Bhushan, O. A. Bég, Analytical study of electro-osmosis modulated capillary peristaltic hemodynamics, J. Mech. Med. Biol. 17 (2017) 1750052(1-22).

[17] T. W. H. Sheu, V. C. Huang, H. P. Rani, Development of an electro-osmotic flow model to study the dynamic behaviour 
in human meridian, Int. J. Numer. Methods Fluids 56 (2008) 739-751.

[18] S. Ghosal, Lubrication theory for electro-osmotic flow in a microfluidic channel of slowly varying cross-section and wall charge, J. Fluid Mechanics 459 (2002) 103-128.

[19] K. Bengtsson, N. D. Robinson, A large-area, all-plastic, flexible electroosmotic pump, Microfluidics and Nanofluidics 21 (2017) 178.

[20] G. Bohme (Ed.), Non-Newtonian Fluid Mechanics.

[21] O. A. Bég, J. Zueco, S. K. Ghosh, Unsteady natural convection of a short-memory viscoelastic fluid in a non-darcian regime: network simulation, Chemical Engineering Communications 198 (2010) 172-190.

[22] D. Tripathi, O. A. Bég, A numerical study of oscillating peristaltic flow of generalized maxwell viscoelastic fluids through a porous medium, Transport in Porous Media 95 (2012) 337-348.

[23] M. Norouzi, M. Davoodi, O. A. Bég, A. A. Joneidi, Analysis of the effect of normal stress differences on heat transfer in creeping viscoelastic dean flow, Int. J. Thermal Sciences 69 (2013) 61-69.

[24] D. Tripathi, O. A. Bég, Mathematical modelling of heat transfer effects on swallowing dynamics of viscoelastic flood bolus through the human oesophagus, Int. J. Thermal Sciences 70 (2013) 41-53.

[25] T. Alarabi, A. F. Elsayed, O. A. Bég, Homotopy perturbation method for heat transfer in peristaltic flow of viscoelastic fluid in an eccentric cylinder with variable effects, Life Science Journal 11(7) (2014) 197-206.

[26] A. Sadeghi, M. H. Saidi, A. A. Mozafari, Heat transfer due to electroosmotic flow of viscoelastic fluids in a slit microchannel, Int. J. Heat Mass Transfer 54 (2011) 4069-4077.

[27] C. L. A. Berli, M. Olivares, Electrokinetic flow of non-newtonian fluids in microchannels, J. Colloid Interface Sci. 320 (2008) 582-589.

[28] L. L. Ferrás, A. M. Afonso, M. A. Alves, J. M. Nóbrega, F.T.Pinho, Analytical and numerical study of the electroosmotic annular flow of viscoelastic fluids, J. Colloid Interface Sci. 420 (2014) 152-157.

[29] Y. Jiang, H. Qi, H. Xu, X. Jiang, Transient electroosmotic slip flow of fractional oldroyd-b fluids, Microfluidics and Nanofluidics 21 (2017) 7.

[30] J. C. Misra, S. Chandra, G. C. Shit, P. K. Kundu, Electroosmotic oscillatory flow of micropolar fluid in microchannels: application to dynamics of blood flow in microfluidic devices, Applied Mathematics and Mechanics 35 (2014) 749-766.

[31] B. J. Kirby, E. F. Hasselbrink, Zeta potential of microfluidic substrates. ii. data for polymers, Electrophoresis 25 (2004) $203-213$.

[32] A. Bandopadhyay, D. Tripathi, S. Chakraborty, Electroosmosis-modulated peristaltic transport in microfluidic channels, Physics of Fluids 28 (2016) 052002(1-24).

[33] D. Huh, B. D. Matthews, A. Mammoto, M. Montoya-Zavala, H. Y. Hsin, D. E. Ingber, Reconstituting organ-level lung functions on a chip, Science 328 (2010) 1662-1668.

[34] H. J. Kim, D. Huh, G. Hamilton, D. E. Ingber, Human gut-on-a-chip inhabited by microbial flora that experiences intestinal peristalsis-like motions and flow, Lab Chip. 12(12) (2012) 2165-2174.

[35] L. Wei-Xuan, L. Guang-Tie, Y. Wei, Z. Qiong, W. Wei, Z. Xiao-Mian, L. Da-Yu, Artificial uterus on a microfluidic 
chip, Chinese Journal of Analytical Chemistry 41(4) (2013) 467-472.

[36] C. Pozrikidis, A study of peristaltic flow, J. Fluid Mech. 180 (1987) 515-527.

[37] S. Yaniv, A. J. Jaffa, D. Elad, Modeling embryo transfer into a closed uterine cavity, J. Biomech. Eng. 134 (2012) $111003(1-7)$.

[38] S. Shaked, A. J. Jaffa, D. Grisaru, D. Elad, Uterine peristalsis-induced stresses within the uterine wall may sprout adenomyosis, Biomech. Model. Mechanobiol. 14(3) (2015) 437-444.

[39] Y. Payan, J. Ohayon, iomechanics of Living Organs: Hyperelastic Constitutive Laws for Finite Element Modeling, Elsevier, 2017. 